\title{
Author Correction: Progress on AlGaN-based solar-blind ultraviolet photodetectors and focal plane arrays
}

Qing Cai $\mathbb{D}$, Haifan You, Hui Guo, Jin Wang, Bin Liu, Zili Xie, Dunjun Chen, Hai Lu, Youdou Zheng and Rong Zhang

Correction to: Light: Science \& Applications

https://doi.org/10.1038/s41377-021-00527-4

published online 30 April 2021

Following publication of the article ${ }^{1}$, it is reported that this article contains some errors. The correction details are listed below.
(1) Figure 19 has been updated as below.

Published online: 07 December 2021

\section{Reference}

1. Cai, Q. et al. Progress on AlGaN-based solar-blind ultraviolet photodetectors and focal plane arrays. Light Sci. Appl. 10, 94 (2021). a

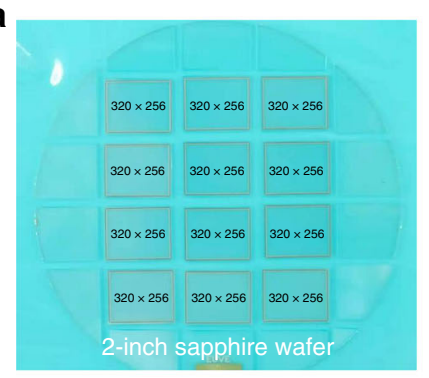

b

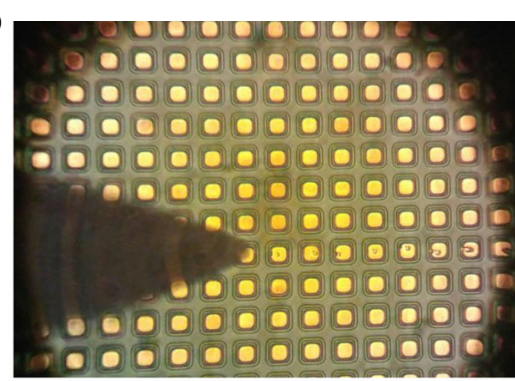

d

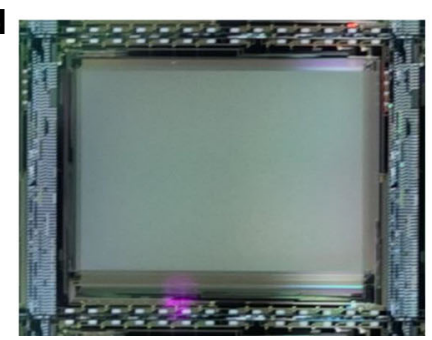

e

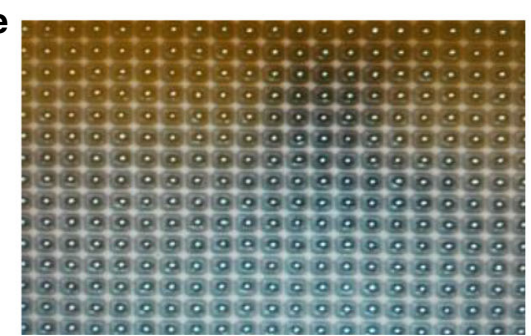

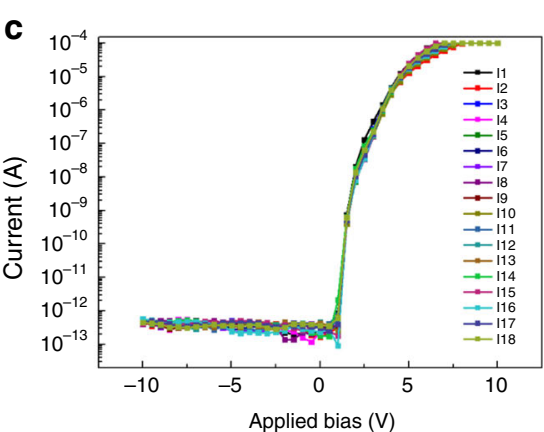

$\mathbf{f}$

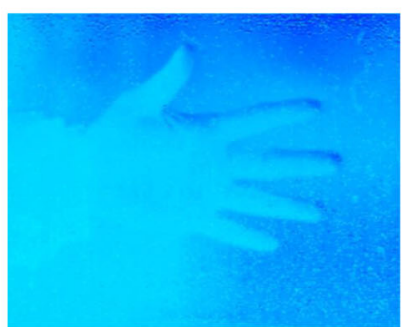

Fig. 19 AIGaN p-i-n solar-blind UV FPAs and imaging by Nanjing University. a $320 \times 256$ AlGaN FPAs on 2-inch sapphire wafer. b Optical images of AIGaN focal plane arrays. c I-V characteristics of the individual photodetector. $\mathbf{d}$ Silicon driver IC. e Optical images of the array with Indium bumps. f Solar-blind ultraviolet images taken from the AIGaN FPA camera

Open Access This article is licensed under a Creative Commons Attribution 4.0 International License, which permits use, sharing, adaptation, distribution and reproduction in any medium or format, as long as you give appropriate credit to the original author(s) and the source, provide a link to the Creative Commons license, and indicate if material is not included in the article's Creative Commons license and your intended use is not permitted by statutory regulation or exceeds the permitted use, you will need to obtain permission directly from the copyright holder. To view a copy of this license, visit http://creativecommons.org/licenses/by/4.0/. 